\title{
A Rare Case of Pulse Granuloma Presenting as Sub-Acute Intestinal Obstruction: Case Report
}

\author{
Ram Babu K ${ }^{1 *}$, Krishna Kanth GVRN ${ }^{2}$, Sudhir Kumar Vujhini ${ }^{3}$ \\ ${ }^{1}$ Senior Specialist, Department of Pathology, ESIC MC Hospital, Sanathnagar, Hyderabad, Telangana, India \\ ${ }^{2}$ Professor, Department of Pathology, ESIC MC Hospital, Sanathnagar, Hyderabad, Telangana, India \\ ${ }^{3}$ Associate Professor, Department of Transfusion medicine, NIMS, Hyderabad, Telangana, India
}

DOI: $\underline{10.36348 / \mathrm{sjpm} .2020 . \mathrm{v} 05 \mathrm{i} 05.013}$

| Received: 14.05.2020 | Accepted: 21.05.2020 | Published: 27.05.2020

*Corresponding author: Ram Babu K

\section{Abstract}

Pulse Granuloma also known as Hyaline Angiopathy is a rare but benign lesion, foreign body reaction to vegetable particle being the probable aetiology. The patient presented with recurrent intestinal obstruction and partial excision of small bowel was performed and specimen sent for histopathological examination. The microscopy showed mixed inflammatory cells, foreign body giant cells, convoluted hyaline rings, vegetable matter and areas of fibrosis. Most of the reported cases are in oral cavity or lungs and very few cases were reported in gastrointestinal tract and still rare in small intestine. Barring low incidence of the lesion, the characteristic histology reveals the lesion which avoids erroneous diagnosis, delay in diagnosis and thus helps in proper management of the case. Treatment of Pulse granuloma is excision. Keywords: Pulse Granuloma, aetiology, histopathological examination.

Copyright @ 2020: This is an open-access article distributed under the terms of the Creative Commons Attribution license which permits unrestricted use, distribution, and reproduction in any medium for non-commercial use (NonCommercial, or CC-BY-NC) provided the original author and sources are credited.

\section{INTRODUCTION}

Pulse granuloma is a rare benign lesion also known as Hyaline angiopathy or known as Oral vegetable granuloma when occurring in the oral cavity. Available literature suggests that the etiopathogenesis is a foreign body giant cell reaction to ingested legume at various stages of its digestion [1, 2]. Extra oral locations so far reported are in lung, gall bladder, fallopian tube, appendix, skin, peritoneum, peridiverticulum, ovary and intra hepatic portal vein [3-6]. To the best of our knowledge this is one of a rare case reported in small intestine.

\section{CASE REPORT}

A 41 yr old female patient presented with recurrent abdominal pain and weight loss. Radiological examination revealed she had sub acute intestinal obstruction involving the ileal and jejunal loops. It also showed ileal loops seen clustered and adherent to the anterior abdominal wall and to each other in right lumbar, iliac fossa, pelvis and right peri-umbilical region, likely due to multiple adhesions in the peritoneum. Retrospective evaluation revealed she was operated for sub acute intestinal obstruction three weeks earlier and the subsequent histopathology showed acute on chronic inflammation of small bowel with accompanying fibrosis. Exploratory laparotomy was performed, adhenolysis done and small bowel 50 centimetres from D-J junction and 20 centimetres from Ileal junction was resected and specimen sent for histopathological examination. Gross examination showed adherent loops of bowel with gangrenous looking distal segment (Figure-1). Cut sections showed thickened bowel walls, intact mucosa of the proximal bowel, adherent bowel loops, presence of vegetable matter in the lumen and gangrenous distal segment. Microscopy showed peri-serosal lesion extending into the adjoining mesenteric fat of ileum with mucosa unaffected to a greater extent. It showed (Figure $2 \& 3$ ). Distal segment showed features of ischemic small bowel amounting to gangrenous change. No specific lesion was considered. But other causes of granulomas like Tuberculosis, fungal were ruled out owing to absence of epithelioid like giant cells and any filamentous hyphae like structures. Parasitic aetiology was also ruled out as the pink hyaline rings which resembled eggs of known helminths measured $200-$ 500 microns, whereas parasite eggs are known to measure less than 100 microns. Intrigued by histology we have reviewed the available literature to categorise this granuloma. It showed existence of Pulse granuloma also known as Hyaline ring granuloma which affects predominantly oral cavity but also know to affect other sites. Past history of segmental resection of small bowel owing to adhesions (subsequent to improper closure of 
peritoneum and injury to the bowel) has believed us to the pathology of this lesion consistent with Pulse granuloma as these granulomas are known to occur in $10 \%$ of injured gastrointestinal tract. Its unique histological features of foreign body giant cells surrounding large convoluted pink hyaline rings and vegetable matter consolidated the diagnosis. PAS (Periodic Acid-Schiff Stain) showed positivity in the hyaline rings and was negative for fungal elements (Figure-4).

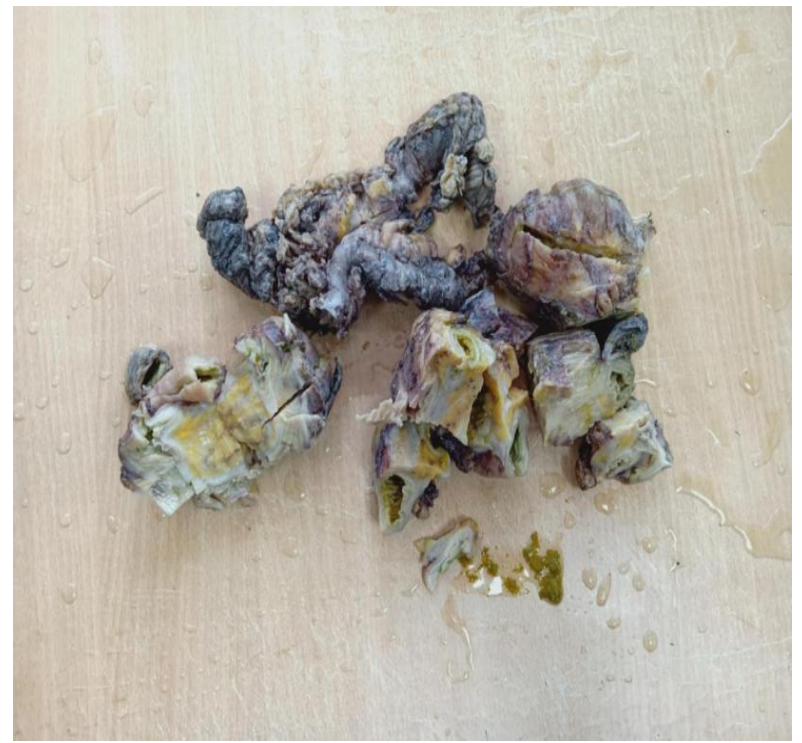

Fig-1: Affected bowel loops with focal gangrenous areas

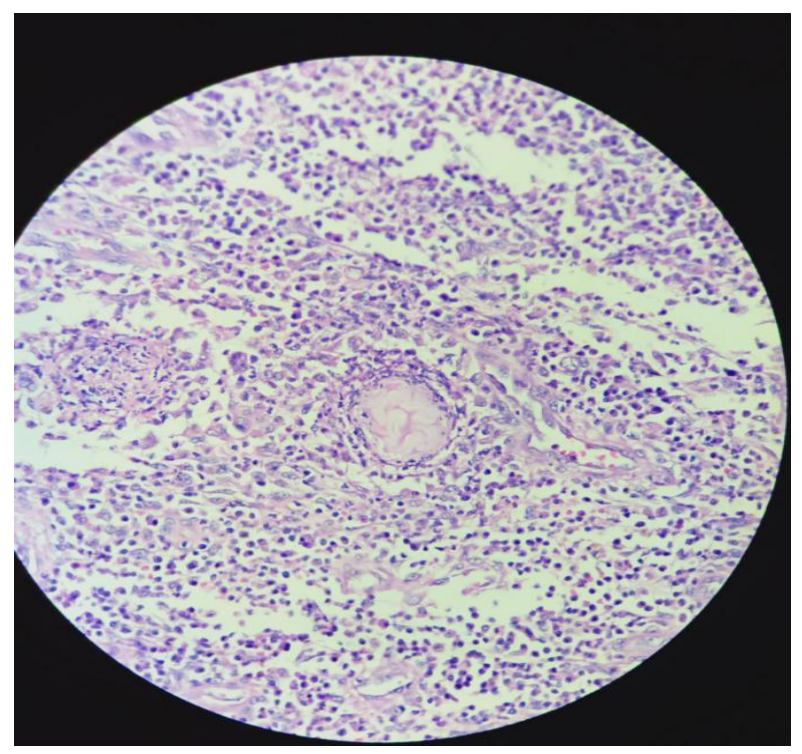

Fig-2: Showing mixed inflammatory cell collection, foreign body giant cells around hyaline ring like structures, vegetable matter and suture material

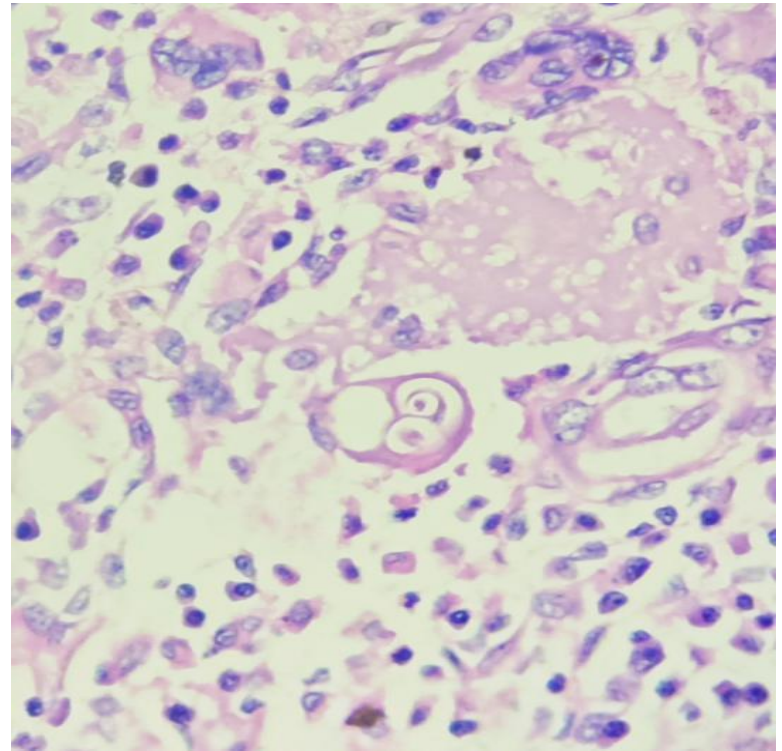

Fig-3: Showing mixed inflammatory cell collection, foreign body giant cells around hyaline ring like structures, vegetable matter and suture material

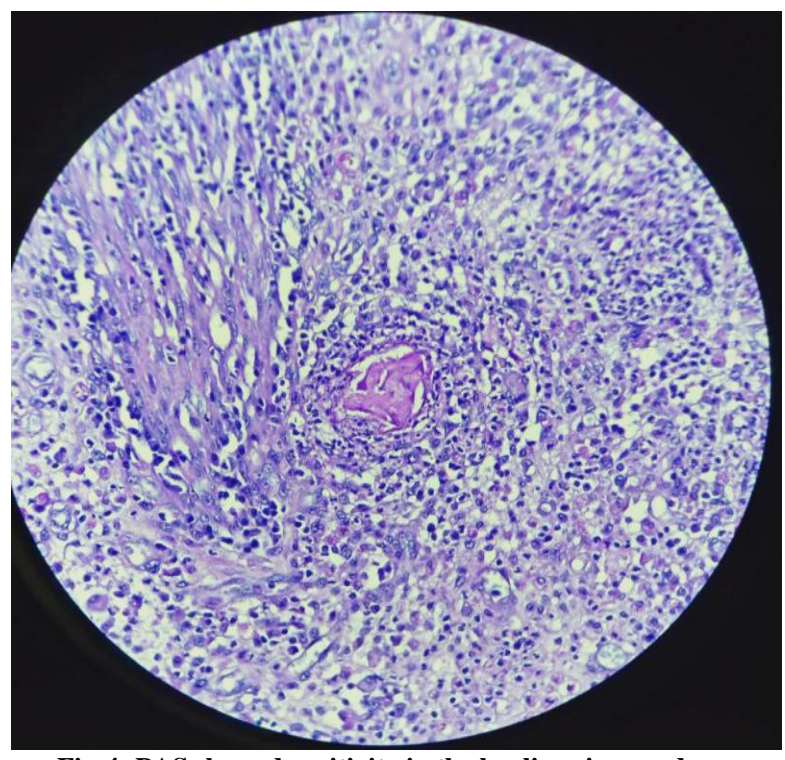

Fig-4: PAS showed positivity in the hyaline rings and was negative for fungal elements

\section{DISCUSSION}

Pulse granulomas are a result of an inflammatory reaction to vegetable matter like peas, lentils and are characterised by presence of mixed inflammatory cell infiltrate, foreign body giant cells and eosinophilic convoluted hyaline rings $[6,8,9]$. Two explanations have been given to possible pathophysiology of Pulse granulomas. Endogenous theory proposes hyaline rings are formed due to degenerative change in blood vessel wall or is a fibrosed extravagated secretory protein [6, 8]. Exogenous theory suggests Pulse granuloma as an inflammatory reaction to the penetration of vegetable matter and these hyaline rings considered to be from partially digested cellulose leguminous material of legumes are noxious and evoke inflammation in contrast to digestible starch of plant food $[1,2]$. Animal 
studies done also provide evidence that exogenous origin is a better hypothesis for formation of Pulse granuloma [2].

Gonzalez recently noted that Pulse granuloma may be noted in $10 \%$ of injured small and large bowel [9]. According to Philipsen HP and Reichart PA for diagnosis of Pulse granuloma following histopathological features need to be present: 1 . Eosinophilic hyaline rings in the form of circular homogenous or fibrillar masses, 2. A characteristic inflammatory lesion, 3. Foreign body giant cells [6, 8].

As is well known granulomas due to diseases such as Crohn's and Sarcoidosis are known to occur in gastro-intestinal tract. Characteristic histological features like crypt abscesses, cryptitis and naked granulomas with asteroid bodies help to exclude these two entities. Absence of epithelioid granulomas, absence of filamentous hyphae like structures with PAS negativity excludes Tuberculosis and Fungal causes. Parasitic aetiology is also ruled out owing to the absence of nuclei in the hyaline rings and there was no morphological conformity with known parasites in our case. The size of hyaline rings resembling parasite eggs was 200-500 microns, which is 2-5 times the size of any known helminthic eggs thus they are ruled out in our case. Post operative adhesions due to glove powder are known to induce granulomatous reaction but these have uniform small round crystalline structures [7]. Hyaline ring structures simulate vascular formations but the elastic and muscular structures of vessels were not present $[1,6]$.

\section{CONCLUSION}

We present a case of Pulse granuloma in small intestine which developed subsequent to an insult to small bowel. Pulse granuloma or Hyaline granuloma is a rare but a well described entity with characteristic histopathological features. Pulse granuloma at sites other than oral region shows on histology more number of giant cells and hyaline rings. Penetration of vegetable matter into an injured site and subsequent inflammatory reaction is the reason for foreign body giant cell reaction leading to Pulse granuloma. The present case emphasises the importance to histopathologist of being acquainted with Pulse granuloma to avoid delay in diagnosis, misdiagnosis and facilitate proper management and follow up.

\section{Financial Support and Sponsorship: Nil}

\section{Conflicts of Interest: None}

\section{REFERENCES}

1. Philipsen, H. P., \& Reichart, P. A. (2010). Pulse or hyaline ring granuloma. Review of the literature on etiopathogenesis of oral and extraoral lesions. Clinical oral investigations, 14(2), 121128.

2. Talacko, A. A., \& Radden, B. G. (1988). The pathogenesis of oral pulse granuloma: an animal model. Journal of Oral Pathology \& Medicine, 17(3), 99-105.

3. Rhee, D. D., \& Wu, M. L. C. (2006). Pulse granulomas detected in gallbladder, fallopian tube, and skin. Archives of pathology \& laboratory medicine, 130(12), 1839-1842.

4. Şımşek, G. G., Buluş, H., \& Güreşç, S. (2012). Pulse granuloma, unusual localization. The Turkish journal of gastroenterology: the official journal of Turkish Society of Gastroenterology, 23(4), 417-418.

5. Karp, J. K., Davis, A., Read, P. J., Mashayekh, A., Bombonati, A., \& Palazzo, F. (2013). Pulse granuloma involving Meckel's diverticulum: a case report and literature review. Pathologica, 105(2), 59-61.

6. Dunlap, C. L., \& Barker, B. F. (1977). Giant-cell hyalin angiopathy. Oral Surgery, Oral Medicine, Oral Pathology, 44(4), 587-591.

7. Davies, J. D., \& Ansell, I. D. (1983). Food-starch granulomatous peritonitis. Journal of clinical pathology, 36(4), 435-438.

8. Chen, S. Y., Fantasia, J. E., \& Miller, A. S. (1981). Hyaline bodies in the connective tissue wall of odontogenic cysts. Journal of Oral Pathology \& Medicine, 10(3), 147-157.

9. Gonzalez, R. S. (2016). Incidence of pulse granuloma in the small and large intestines. The American journal of surgical pathology, 40(1), 137-140. 